\title{
On Growth of Meromorphic Solutions of Complex Functional Difference Equations
}

\author{
Jing Li, ${ }^{1,2}$ Jianjun Zhang, ${ }^{3}$ and Liangwen Liao ${ }^{1}$ \\ ${ }^{1}$ Department of Mathematics, Nanjing University, Nanjing 210093, China \\ ${ }^{2}$ Nankai University Binhai College, Tianjin 300270, China \\ ${ }^{3}$ Mathematics and Information Technology School, Jiangsu Second Normal University, Nanjing 210013, China
}

Correspondence should be addressed to Jianjun Zhang; zhangjianjun1982@163.com

Received 29 November 2013; Accepted 13 January 2014; Published 25 February 2014

Academic Editor: Zong-Xuan Chen

Copyright (C) 2014 Jing Li et al. This is an open access article distributed under the Creative Commons Attribution License, which permits unrestricted use, distribution, and reproduction in any medium, provided the original work is properly cited.

\begin{abstract}
The main purpose of this paper is to investigate the growth order of the meromorphic solutions of complex functional difference equation of the form $\left(\sum_{\lambda \in I} \alpha_{\lambda}(z)\left(\prod_{\nu=1}^{n} f\left(z+c_{\nu}\right)^{l_{\lambda, \nu}}\right)\right) /\left(\sum_{\mu \in J} \beta_{\mu}(z)\left(\prod_{\nu=1}^{n} f\left(z+c_{\nu}\right)^{m_{\mu, v}}\right)\right)=Q(z, f(p(z)))$, where $I=\{\lambda=$ $\left.\left(l_{\lambda, 1}, l_{\lambda, 2}, \ldots, l_{\lambda, n}\right) \mid l_{\lambda, v} \in \mathbb{N} \bigcup\{0\}, v=1,2, \ldots, n\right\}$ and $J=\left\{\mu=\left(m_{\mu, 1}, m_{\mu, 2}, \ldots, m_{\mu, n}\right) \mid m_{\mu, v} \in \mathbb{N} \bigcup\{0\}, v=1,2, \ldots, n\right\}$ are two finite index sets, $c_{\nu}(\nu=1,2, \ldots, n)$ are distinct complex numbers, $\alpha_{\lambda}(z)(\lambda \in I)$ and $\beta_{\mu}(z)(\mu \in J)$ are small functions relative to $f(z)$, and $Q(z, u)$ is a rational function in $u$ with coefficients which are small functions of $f(z), p(z)=p_{k} z^{k}+p_{k-1} z^{k-1}+\cdots+p_{0} \in \mathbb{C}[z]$ of degree $k \geq 1$. We also give some examples to show that our results are sharp.
\end{abstract}

\section{Introduction and Main Results}

Let $f(z)$ be a function meromorphic in the complex plane $\mathbb{C}$. We assume that the reader is familiar with the standard notations and results in Nevanlinna's value distribution theory of meromorphic functions such as the characteristic function $T(r, f)$, proximity function $m(r, f)$, counting function $N(r, f)$, and the first and second main theorems (see, e.g., [1$4])$. We also use $\bar{N}(r, f)$ to denote the counting function of the poles of $f(z)$ whose every pole is counted only once. The notations $\rho(f)$ and $\mu(f)$ denote the order and the lower order of $f(z)$, respectively. $S(r, f)$ denotes any quantity that satisfies the condition: $S(r, f)=o(T(r, f))$ as $r \rightarrow \infty$ possibly outside an exceptional set of $r$ of finite linear measure. A meromorphic function $a(z)$ is called a small function of $f(z)$ or a small function relative to $f(z)$ if and only if $T(r, a(z))=$ $S(r, f)$.

Recently, some papers (see, e.g., [5-7]) focusing on complex difference and functional difference equations emerged. In 2005, Laine et al. [5] firstly considered the growth of meromorphic solutions of the complex functional difference equations by utilizing Nevanlinna theory. They obtained the following result.
Theorem A. Suppose that $f$ is a transcendental meromorphic solution of the equation

$$
\sum_{\{J\}} \alpha_{J}(z)\left(\prod_{j \in J} f\left(z+c_{j}\right)\right)=f(p(z)),
$$

where $\{J\}$ is a collection of all subsets of $\{1,2, \ldots, n\}, c_{j}$ 's are distinct complex constants, and $p(z)$ is a polynomial of degree $k \geq 2$. Moreover, we assume that the coefficients $\alpha_{J}(z)$ are small functions relative to $f$ and that $n \geq k$. Then

$$
T(r, f)=O\left((\log r)^{\alpha+\varepsilon}\right),
$$

where $\alpha=\log n / \log k$.

In 2007, Rieppo [6] gave an estimation of growth of meromorphic solutions of complex functional equations as follows.

Theorem B. Suppose that $f$ is a transcendental meromorphic function. Let $Q(z, f), R(z, f)$ be rational functions in $f$ with small meromorphic coefficients relative to $f$ such that $0<q:=\operatorname{deg}_{f} Q \leq d:=\operatorname{deg}_{f} R$ and $p(z)=p_{k} z^{k}+p_{k-1} z^{k-1}+$ 
$\cdots+p_{0} \in \mathbb{C}[z]$ of degree $k>1$. If $f$ is a solution of the functional equation

$$
R(z, f(z))=Q(z, f(p(z))),
$$

then $q k \leq d$, and for any $\varepsilon, 0<\varepsilon<1$, there exist positive real constants $K_{1}$ and $K_{2}$ such that

$$
K_{1}(\log r)^{\alpha-\varepsilon} \leq T(r, f) \leq K_{2}(\log r)^{\alpha+\varepsilon}, \quad \alpha=\frac{\log d-\log q}{\log k},
$$

when $r$ is large enough.

Rieppo [6] also considered the growth order of meromorphic solutions of functional equation (3) when $k=1$ and got the following.

Theorem C. Suppose that $f$ is a transcendental meromorphic solution of (3), where $p(z)=a z+b, a, b \in \mathbb{C}, a \neq 0$ and $|a| \neq 1$. Then

$$
\mu(f)=\rho(f)=\frac{\log d-\log q}{\log |a|} .
$$

Two years later, Zheng et al. [7] extended Theorem A to more general type and obtained a similar result of Theorem C. In fact, they got the following two results.

Theorem D. Suppose that $f$ is a transcendental meromorphic solution of the equation

$$
\sum_{\{J\}} \alpha_{J}(z)\left(\prod_{j \in J} f\left(z+c_{j}\right)\right)=Q(z, f(p(z))),
$$

where $\{J\}$ is a collection of all nonempty subsets of $\{1,2, \ldots, n\}$, $c_{j}(j=1, \ldots, n)$ are distinct complex constants, $p(z)=p_{k} z^{k}+$ $p_{k-1} z^{k-1}+\cdots+p_{0} \in \mathbb{C}[z]$ of degree $k>1$, and $Q(z, u)$ is a rational function in $u$ of $\operatorname{deg}_{u} Q=q(>0)$. Also suppose that all the coefficients of (6) are small functions relative to $f$. Then $q k \leq n$, and

$$
T(r, f)=O\left((\log r)^{\alpha+\varepsilon}\right),
$$

where $\alpha=(\log n-\log q) / \log k$.

Theorem E. Suppose that $f$ is a transcendental meromorphic solution of (6), where $\{J\}$ is a collection of all nonempty subsets of $\{1,2, \ldots, n\}, c_{j}(j=1, \ldots, n)$ are distinct complex constants, $p(z)=a z+b, a, b \in \mathbb{C}$, and $Q(z, u)$ is a rational function in $u$ of $\operatorname{deg}_{u} Q=q(>0)$. Also suppose that all the coefficients of (6) are small functions relative to $f$.

(i) If $0<|a|<1$, then we have

$$
\mu(f) \geq \frac{\log q-\log n}{-\log |a|} .
$$

(ii) If $|a|>1$, then we have $q \leq n$ and

$$
\rho(f) \leq \frac{\log n-\log q}{\log |a|} .
$$

(iii) If $|a|=1, q>n$, then we have $\rho(f)=\mu(f)=\infty$.
In this paper, we will consider a more general class of complex functional difference equations. We prove the following results, which generalize the above related results.

Theorem 1. Suppose that $f(z)$ is a transcendental meromorphic solution of the functional difference equation

$$
\frac{\sum_{\lambda \in I} \alpha_{\lambda}(z)\left(\prod_{\nu=1}^{n} f\left(z+c_{\nu}\right)^{l_{\lambda, \nu}}\right)}{\sum_{\mu \in J} \beta_{\mu}(z)\left(\prod_{\nu=1}^{n} f\left(z+c_{\nu}\right)^{m_{\mu, \nu}}\right)}=Q(z, f(p(z))),
$$

where $c_{v}(\nu=1, \ldots, n)$ are distinct complex constants, $I=\{\lambda=$ $\left.\left(l_{\lambda, 1}, l_{\lambda, 2}, \ldots, l_{\lambda, n}\right) \mid l_{\lambda, v} \in \mathbb{N} \bigcup\{0\}, \nu=1,2, \ldots, n\right\}$ and $J=$ $\left\{\mu=\left(m_{\mu, 1}, m_{\mu, 2}, \ldots, m_{\mu, n}\right) \mid m_{\mu, \nu} \in \mathbb{N} \bigcup\{0\}, v=1,2, \ldots, n\right\}$ are two finite index sets, $p(z)=p_{k} z^{k}+p_{k-1} z^{k-1}+\cdots+p_{0} \in$ $\mathbb{C}[z]$ of degree $k>1$, and $Q(z, u)$ is a rational function in $u$ of $\operatorname{deg}_{u} Q=q(>0)$. Also suppose that all the coefficients of (10) are small functions relative to $f$. Denoting

$$
\sigma_{\nu}=\max _{\lambda, \mu}\left\{l_{\lambda, \nu}, m_{\mu, \nu}\right\} \quad(\nu=1,2, \ldots, n), \sigma=\sum_{\nu=1}^{n} \sigma_{\nu} .
$$

Then $q k \leq \sigma$, and

$$
T(r, f)=O\left((\log r)^{\alpha+\varepsilon}\right)
$$

where $\alpha=(\log \sigma-\log q) / \log k$.

Theorem 2. Suppose that $f$ is a transcendental meromorphic solution of the equation

$$
\frac{\sum_{\lambda \in I} \alpha_{\lambda}(z)\left(\prod_{\nu=1}^{n} f\left(z+c_{\nu}\right)^{l_{\lambda, \nu}}\right)}{\sum_{\mu \in J} \beta_{\mu}(z)\left(\prod_{\nu=1}^{n} f\left(z+c_{\nu}\right)^{m_{\mu, \nu}}\right)}=Q(z, f(a z+b))
$$

where $c_{\nu}(\nu=1, \ldots, n)$ are distinct complex constants, $I=\{\lambda=$ $\left.\left(l_{\lambda, 1}, l_{\lambda, 2}, \ldots, l_{\lambda, n}\right) \mid l_{\lambda, v} \in \mathbb{N} \bigcup\{0\}, \nu=1,2, \ldots, n\right\}$ and $J=\{\mu=$ $\left.\left(m_{\mu, 1}, m_{\mu, 2}, \ldots, m_{\mu, n}\right) \mid m_{\mu, \nu} \in \mathbb{N} \bigcup\{0\}, v=1,2, \ldots, n\right\}$ are two finite index sets, $a, b \in \mathbb{C}$, and $Q(z, u)$ is a rational function in $u$ of $\operatorname{deg}_{u} Q=q(>0)$. Also suppose that all the coefficients of (10) are small functions relative to $f$. Denoting

$$
\sigma_{\nu}=\max _{\lambda, \mu}\left\{l_{\lambda, \nu}, m_{\mu, \nu}\right\} \quad(\nu=1,2, \ldots, n), \sigma=\sum_{\nu=1}^{n} \sigma_{\nu} .
$$

(i) If $0<|a|<1$, then we have

$$
\mu(f) \geq \frac{\log q-\log \sigma}{-\log |a|} .
$$

(ii) If $|a|>1$, then we have $q \leq \sigma$ and

$$
\rho(f) \leq \frac{\log \sigma-\log q}{\log |a|} .
$$

(iii) If $|a|=1$ and $q>\sigma$, then we have $\mu(f)=\rho(f)=\infty$. 
Next we will give some examples to show that our results are best in some extent.

Example 3. Let $c_{1}=\arctan 2, c_{2}=-\pi / 4$. Then it is easy to check that $f(z)=\tan z$ solves the following equation:

$$
\begin{aligned}
& \frac{f\left(z+c_{1}\right)^{2} f\left(z+c_{2}\right)}{f\left(z+c_{1}\right)+f\left(z+c_{2}\right)^{2}} \\
& =\left(-4 f\left(\frac{z}{2}\right)^{8}+8 f\left(\frac{z}{2}\right)^{7}+28 f\left(\frac{z}{2}\right)^{6}-56 f\left(\frac{z}{2}\right)^{5}\right. \\
& \left.\quad-32 f\left(\frac{z}{2}\right)^{4}+56 f\left(\frac{z}{2}\right)^{3}+28 f\left(\frac{z}{2}\right)^{2}-8 f\left(\frac{z}{2}\right)-4\right) \\
& \times\left(3 f\left(\frac{z}{2}\right)^{8}+10 f\left(\frac{z}{2}\right)^{7}+16 f\left(\frac{z}{2}\right)^{6}+122 f\left(\frac{z}{2}\right)^{5}\right. \\
& \left.\quad-6 f\left(\frac{z}{2}\right)^{4}-122 f\left(\frac{z}{2}\right)^{3}+16 f\left(\frac{z}{2}\right)^{2}-10 f\left(\frac{z}{2}\right)+3\right)^{-1} .
\end{aligned}
$$

Obviously, we have

$$
\mu(f)=\rho(f)=1=\frac{\log q-\log \sigma}{-\log |a|},
$$

where $q=8, \sigma=4$ and $a=1 / 2$. sharp.

Example 3 shows that the estimate in Theorem 2(i) is

Example 4. It is easy to check that $f(z)=\tan z$ satisfies the equation

$$
\begin{gathered}
\frac{f(z+(\pi / 3))^{2} f(z+(\pi / 6))-f(z+(\pi / 6))}{f(z+(\pi / 3)) f(z+(\pi / 6))^{2}-f(z+(\pi / 3))} \\
=\frac{\sqrt{3} f(2 z)^{2}+4 f(2 z)+\sqrt{3}}{-\sqrt{3} f(2 z)^{2}+4 f(2 z)-\sqrt{3}} .
\end{gathered}
$$

Clearly, we have

$$
\mu(f)=\rho(f)=1=\frac{\log \sigma-\log q}{\log |a|},
$$

where $\sigma=4, q=2$ and $a=2$. sharp.

Example 4 shows that the estimate in Theorem 2(ii) is

Example 5. $f(z)=\tan z$ satisfies the equation of the form

$$
\begin{aligned}
& \frac{f(z+(\pi / 4))^{2}}{f(z+(\pi / 4))+f(z-(\pi / 4))^{2}} \\
& =\frac{-\left(f(z / 2)^{2}-2 f(z / 2)-1\right)^{3}}{8 f(z / 2)\left(f(z / 2)^{2}-1\right)\left(f(z / 2)^{2}+2 f(z / 2)-1\right)},
\end{aligned}
$$

where $\sigma=4, q=6$, and $a=1 / 2 . \rho(f)=\mu(f)=1>$ $\log (3 / 2) / \log 2=(\log q-\log \sigma) /-\log |a|$.
Example 5 shows that the strict inequality in Theorem 2 may occur. Therefore, we do not have the same estimation as in Theorem $\mathrm{C}$ for the growth order of meromorphic solutions of (13).

The following Example shows that the restriction $q>\sigma$ in case (iii) in Theorem 2 is necessary.

Example 6. Meromorphic function $f(z)=\tan z$ solves the following equation:

$$
\frac{f(z+(\pi / 4))^{2}}{f(z+(\pi / 4))+f(z-(\pi / 4))^{2}}=\frac{(f(z)+1)^{3}}{4 f(z)(1-f(z))},
$$

where $a=1$ and $4=\sigma>q=3$, but $\rho(f)=\mu(f)=1$.

Next, we give an example to show that case (iii) in Theorem 2 may hold.

Example 7. Function $f(z)=z e^{e^{z}}$ satisfies the following equation:

$$
\begin{gathered}
\frac{(z+\log 6)(z+\log 2)^{5}\left[f(z+\log 4)^{4}+f(z+\log 4)\right]}{(z+\log 4) f(z+\log 6)} \\
=\frac{(z+\log 4)^{3} f(z+\log 2)^{6}+(z+\log 2)^{6}}{f(z+\log 2)},
\end{gathered}
$$

where $a=1$ and $q=6>5=\sigma$. Obviously, $\rho(f)=\mu(f)=\infty$.

\section{Main Lemmas}

In order to prove our results, we need the following lemmas.

Lemma 1 (see $[4,8]$ ). Let $f(z)$ be a meromorphic function. Then for all irreducible rational functions in $f$,

$$
R(z, f)=\frac{P(z, f)}{Q(z, f)}=\frac{\sum_{i=0}^{p} a_{i}(z) f^{i}}{\sum_{j=0}^{q} b_{j}(z) f^{j}},
$$

such that the meromorphic coefficients $a_{i}(z), b_{j}(z)$ satisfy

$$
\begin{aligned}
& T\left(r, a_{i}\right)=S(r, f), \quad i=0,1, \ldots, p, \\
& T\left(r, b_{j}\right)=S(r, f), \quad j=0,1, \ldots, q ;
\end{aligned}
$$

then one has

$$
T(r, R(z, f))=\max \{p, q\} \cdot T(r, f)+S(r, f) .
$$

From the proof of Theorem 1 in [9], we have the following estimate for the Nevanlinna characteristic.

Lemma 2. Let $f_{1}, f_{2}, \ldots, f_{n}$ be distinct meromorphic functions and

$$
F(z)=\frac{P(z)}{Q(z)}=\frac{\sum_{\lambda \in I} \alpha_{\lambda}(z) f_{1}^{l_{\lambda, 1}} f_{2}^{l_{\lambda, 2}} \ldots f_{n}^{l_{\lambda, n}}}{\sum_{\mu \in J} \beta_{\mu}(z) f_{1}^{m_{\mu, 1}} f_{2}^{m_{\mu, 2}} \ldots f_{n}^{m_{\mu, n}}} .
$$


Then

$$
T(r, F(z)) \leq \sum_{\nu=1}^{n} \sigma_{\nu} T\left(r, f_{\nu}\right)+S(r, f),
$$

where $I=\left\{\lambda=\left(l_{\lambda, 1}, l_{\lambda, 2}, \ldots, l_{\lambda, n}\right) \mid l_{\lambda, v} \in \mathbb{N} \bigcup\{0\}, v=\right.$ $1,2, \ldots, n\}$ and $J=\left\{\mu=\left(m_{\mu, 1}, m_{\mu, 2}, \ldots, m_{\mu, n}\right) \mid m_{\mu, v} \in\right.$ $\mathbb{N} \bigcup\{0\}, \nu=1,2, \ldots, n\}$ are two finite index sets, $\sigma_{v}=$ $\max _{\lambda, \mu}\left\{l_{\lambda, \nu}, m_{\mu, \nu}\right\}(\nu=1,2, \ldots, n) . \alpha_{\lambda}(z)=o\left(T\left(r, f_{\nu}\right)(\lambda \in I)\right)$ and $\beta_{\mu}(z)=o\left(T\left(r, f_{\nu}\right)(\mu \in J)\right)$ hold for all $\nu \in\{1,2, \ldots, n\}$ and satisfy $T\left(r, \alpha_{\lambda}\right)=S(r, f)(\lambda \in I)$ and $T\left(r, \beta_{\mu}\right)=S(r, f)(\mu \in$ $J)$.

Lemma 3 (see [7]). Let c be a complex constant. Given $\varepsilon>0$ and a meromorphic function $f$, one has

$$
T(r, f(z \pm c)) \leq(1+\varepsilon) T(r+|c|, f),
$$

for all $r>r_{0}$, where $r_{0}$ is some positive constant.

Lemma 4 (see $[4])$. Let $g:(0,+\infty) \rightarrow \mathbb{R}, h:(0,+\infty) \rightarrow \mathbb{R}$ be monotone increasing functions such that $g(r) \leq h(r)$ outside of an exceptional set $E$ of finite linear measure. Then, for any $\alpha>1$, there exists $r_{0}>0$ such that $g(r) \leq h(\alpha r)$ for all $r>r_{0}$.

Lemma 5 (see [10]). Let $f$ be a transcendental meromorphic function, and $p(z)=a_{k} z^{k}+a_{k-1} z^{k-1}+\cdots+a_{1} z+a_{0}, a_{k} \neq 0$, be a nonconstant polynomial of degree $k$. Given $0<\delta<\left|a_{k}\right|$, denote $\lambda=\left|a_{k}\right|+\delta$ and $\mu=\left|a_{k}\right|-\delta$. Then given $\varepsilon>0$ and $a \in \mathbb{C} \bigcup\{\infty\}$, one has

$$
\begin{gathered}
k n\left(\mu r^{k}, a, f\right) \leq n(r, a, f(p(z))) \leq k n\left(\lambda r^{k}, a, f\right) \\
N\left(\mu r^{k}, a, f\right)+O(\log r) \leq N(r, a, f(p(z))) \\
\leq N\left(\lambda r^{k}, a, f\right)+O(\log r) \\
(1-\varepsilon) T\left(\mu r^{k}, f\right) \leq T(r, f(p(z))) \leq(1+\varepsilon) T\left(\lambda r^{k}, f\right),
\end{gathered}
$$

for all $r$ large enough.

Lemma 6 (see [11]). Let $\phi:\left[r_{0},+\infty\right) \rightarrow(0,+\infty)$ be positive and bounded in every finite interval, and suppose that $\phi\left(\mu r^{m}\right) \leq A \phi(r)+B$ holds for all $r$ large enough, where $\mu>0$, $m>1, A>1$ and $B$ are real constants. Then

$$
\phi(r)=O\left((\log r)^{\alpha}\right),
$$

where $\alpha=\log A / \log m$.

Lemma 7 (see [6]). Let $\phi:\left(r_{0}, \infty\right) \rightarrow(1, \infty)$, where $r_{0} \geq 1$, be a monotone increasing function. If for some real constant $\alpha>1$, there exists a real number $K>1$ such that $\phi(\alpha r) \geq$ $K \phi(r)$, then

$$
\varliminf_{r \rightarrow \infty} \frac{\log \phi(r)}{\log r} \geq \frac{\log K}{\log \alpha} .
$$

Lemma 8 (see $[12])$. Let $\phi:(1, \infty) \rightarrow(0, \infty)$ be a monotone increasing function and let $f$ be a nonconstant meromorphic function. If, for some real constant $\alpha \in(0,1)$, there exist real constants $K_{1}>0$ and $K_{2} \geq 1$ such that

$$
T(r, f) \leq K_{1} \phi(\alpha r)+K_{2} T(\alpha r, f)+S(\alpha r, f),
$$

then

$$
\rho(f) \leq \frac{\log K_{2}}{-\log \alpha}+\varlimsup_{r \rightarrow \infty} \frac{\log \phi(r)}{\log r} .
$$

\section{Proof of Theorems}

Proof of Theorem 1. We assume $f(z)$ is a transcendental meromorphic solution of (10). Denoting $C=$ $\max \left\{\left|c_{1}\right|,\left|c_{2}\right|, \ldots,\left|c_{n}\right|\right\}$. According to Lemmas 1,2 , and 3 and the last assertion of Lemma 5 , we get that for any $\varepsilon_{1}>0$,

$$
\begin{aligned}
q(1- & \left.\varepsilon_{1}\right) T\left(\mu r^{k}, f\right)+S(r, f) \\
& \leq q T(r, f(p(z)))+S(r, f) \\
& =T(r, Q(z, f(p(z)))) \\
& =T\left(r, \frac{\sum_{\lambda \in I} \alpha_{\lambda}(z)\left(\prod_{\nu=1}^{n} f\left(z+c_{\nu}\right)^{l_{\lambda, \nu}}\right)}{\sum_{\mu \in J} \beta_{\mu}(z)\left(\prod_{\nu=1}^{n} f\left(z+c_{\nu}\right)^{m_{\mu, \nu}}\right)}\right) \\
& \leq \sum_{\nu=1}^{n} \sigma_{\nu} T\left(r, f\left(z+c_{\nu}\right)\right)+S(r, f) \\
& \leq \sum_{\nu=1}^{n} \sigma_{\nu}\left(1+\varepsilon_{1}\right) T(r+C, f(z))+S(r, f) \\
& =\left(\sum_{\nu=1}^{n} \sigma_{\nu}\right)\left(1+\varepsilon_{1}\right) T(r+C, f(z))+S(r, f) \\
& =\sigma\left(1+\varepsilon_{1}\right) T(r+C, f(z))+S(r, f),
\end{aligned}
$$

where $r$ is large enough and $\mu=\left|p_{k}\right|-\delta$ for some $0<\delta<\left|p_{k}\right|$. Since $T(r+C, f) \leq T(\beta r, f)$ holds for $r$ large enough for $\beta>1$, we may assume $r$ to be large enough to satisfy

$$
q\left(1-\varepsilon_{1}\right) T\left(\mu r^{k}, f\right) \leq \sigma\left(1+\varepsilon_{1}\right) T(\beta r, f)
$$

outside a possible exceptional set of finite linear measure. By Lemma 4, we know that whenever $\gamma>1$,

$$
q\left(1-\varepsilon_{1}\right) T\left(\mu r^{k}, f\right) \leq \sigma\left(1+\varepsilon_{1}\right) T(\gamma \beta r, f)
$$

holds for all $r$ large enough. Denote $t=\gamma \beta r$; thus the inequality (37) may be written in the form

$$
T\left(\frac{\mu}{(\gamma \beta)^{k}} t^{k}, f\right) \leq \frac{\sigma\left(1+\varepsilon_{1}\right)}{q\left(1-\varepsilon_{1}\right)} T(t, f) .
$$

By Lemma 6, we have

$$
T(r, f)=O\left((\log r)^{\alpha_{1}}\right)
$$


where

$$
\begin{aligned}
\alpha_{1} & =\frac{\log \left(\sigma\left(1+\varepsilon_{1}\right) / q\left(1-\varepsilon_{1}\right)\right)}{\log k} \\
& =\frac{\log \sigma-\log q}{\log k}+\frac{\log \left(\left(1+\varepsilon_{1}\right) /\left(1-\varepsilon_{1}\right)\right)}{\log k} .
\end{aligned}
$$

Denoting now $\alpha=(\log \sigma-\log q) / \log k$ and $\varepsilon=\log ((1+$ $\left.\left.\varepsilon_{1}\right) /\left(1-\varepsilon_{1}\right)\right) / \log k$; thus we obtain the required form.

Finally, we show that $q k \leq \sigma$. If $q k>\sigma$, then we have $\alpha<1$. For sufficiently small $\varepsilon>0$, we have $\alpha+\varepsilon<1$, which contradicts with the transcendency of $f$. Thus Theorem 1 is proved.

Proof of Theorem 2. Suppose $f(z)$ is a transcendental meromorphic solution of (13). Denoting $C=\max \left\{\left|c_{1}\right|,\left|c_{2}\right|\right.$, $\left.\ldots,\left|c_{n}\right|\right\}$.

(i) $0<|a|<1$. We may assume that $q>\sigma$, since the case $q \leq \sigma$ is trivial by the fact that $\mu(f) \geq 0$. By Lemmas $1-3$, we have for any $\varepsilon>0$ and $\beta>1$,

$$
\begin{aligned}
q T(r, & f(p(z)))+S(r, f) \\
& =T(r, Q(z, f(p(z)))) \\
& =T\left(r, \frac{\sum_{\lambda \in I} \alpha_{\lambda}(z)\left(\prod_{\nu=1}^{n} f\left(z+c_{\nu}\right)^{l_{\lambda, v}}\right)}{\sum_{\mu \in J} \beta_{\mu}(z)\left(\prod_{\nu=1}^{n} f\left(z+c_{\nu}\right)^{m_{\mu, \nu}}\right)}\right) \\
& \leq \sum_{\nu=1}^{n} \sigma_{\nu} T\left(r, f\left(z+c_{\nu}\right)\right)+S(r, f) \\
& \leq \sum_{\nu=1}^{n} \sigma_{\nu}(1+\varepsilon) T(r+C, f(z))+S(r, f) \\
& =\left(\sum_{\nu=1}^{n} \sigma_{\nu}\right)(1+\varepsilon) T(r+C, f(z))+S(r, f) \\
& =\sigma(1+\varepsilon) T(r+C, f(z))+S(r, f) \\
& \leq \sigma(1+\varepsilon) T(\beta r, f)+S(r, f),
\end{aligned}
$$

where $r$ is large enough.

By the last assertion of Lemma 5 and (41), we obtain that, for $\mu=|a|-\delta(0<\delta<|a|, 0<\mu<1)$, the following inequality

$$
q(1-\varepsilon) T(\mu r, f) \leq \sigma(1+\varepsilon) T(\beta r, f)
$$

holds, where $r$ is large enough outside of a possible set of finite linear measure. By Lemma 4, we get that for any $\gamma>1$ and sufficiently large $r$,

$$
q(1-\varepsilon) T(\mu r, f) \leq \sigma(1+\varepsilon) T(\gamma \beta r, f) .
$$

Therefore,

$$
\frac{q(1-\varepsilon)}{\sigma(1+\varepsilon)} T(r, f) \leq T\left(\frac{\gamma \beta}{\mu} r, f\right) .
$$

Since $\beta>1, \gamma>1,0<\mu<1$ and $q>\sigma$, we have $\beta \gamma / \mu>1$ and $q(1-\varepsilon) / \sigma(1+\varepsilon)>1$ when $\varepsilon$ is small enough. Using Lemma 7, we see that

$$
\mu(f) \geq \frac{\log q(1-\varepsilon)-\log \sigma(1+\varepsilon)}{\log \gamma \beta-\log \mu} .
$$

Letting $\varepsilon \rightarrow 0, \delta \rightarrow 0, \beta \rightarrow 1$ and $\gamma \rightarrow 1$, we have

$$
\mu(f) \geq \frac{\log q-\log \sigma}{-\log |a|} .
$$

(ii) $|a|>1$. By the similar reasoning as is (i), we easily obtain that

$$
\begin{aligned}
& q(1-\varepsilon) T(\mu r, f) \leq q T(r, f(p(z))) \\
& \quad \leq \sigma(1+\varepsilon) T(r+C, f(z))+S(r, f)
\end{aligned}
$$

for all $r$ large enough. We may select sufficiently small numbers $\delta>0$ and $\varepsilon>0$, such that $\mu=|a|-\delta>1$ and $(1 / \mu)+\varepsilon<1$. Thus we have

$$
T(\mu r, f) \leq \frac{\sigma(1+\varepsilon)}{q(1-\varepsilon)} T(r+C, f(z))+S(r, f) ;
$$

namely,

$$
T(\mu r, f) \leq \frac{\sigma(1+\varepsilon)}{q(1-\varepsilon)} T(r+C, f(z))
$$

where $r$ is large enough possibly outside of a set of finite linear measure. By Lemma 4, we have for any $1<\gamma<\mu$,

$$
T(\mu r, f) \leq \frac{\sigma(1+\varepsilon)}{q(1-\varepsilon)} T(\gamma r, f(z)) ;
$$

that is,

$$
T(r, f) \leq \frac{\sigma(1+\varepsilon)}{q(1-\varepsilon)} T\left(\frac{\gamma}{\mu} r, f(z)\right)
$$

holds for all sufficiently large $r$. By Lemma 8 , we obtain

$$
\rho(f) \leq \frac{\log \sigma-\log q+\log (1+\varepsilon)-\log (1-\varepsilon)}{-\log (\gamma / \mu)} .
$$

Letting $\varepsilon \rightarrow 0, \delta \rightarrow 0$ and $\gamma \rightarrow 1$, we have

$$
\rho(f) \leq \frac{\log \sigma-\log q}{\log |a|} .
$$

(iii) $|a|=1$ and $q>\sigma$. The proof of this case is completely similar as in the case in (i). In fact, we set $\mu=|a|-\delta=$ $1-\delta(0<\delta<1,0<\mu<1)$. Similarly, we can get

$$
\mu(f) \geq \frac{\log q-\log \sigma}{-\log |a|} .
$$

Since $|a|=1$, we have $\mu(f)=\rho(f)=\infty$. 


\section{Conflict of Interests}

The authors declare that there is no conflict of interests regarding the publication of this paper.

\section{Acknowledgments}

The authors would like to thank the anonymous referees for their valuable comments and suggestions. The research was supported by Colonel-level topics (JSNU-ZY01), (Jsie2012zd01), and NSF of China (11271179).

\section{References}

[1] W. Cherry and Z. Ye, Nevanlinna's Theory of Value Distribution, Springer Monographs in Mathematics, Springer, Berlin, Germany, 2001.

[2] W. K. Hayman, Meromorphic Functions, Oxford Mathematical Monographs, Clarendon Press, Oxford, UK, 1964.

[3] Y. Z. He and X. Z. Xiao, Algebroid Functions and Ordinary Differential Equations, Beijing, China, 1988.

[4] I. Laine, Nevanlinna Theory and Complex Differential Equations, vol. 15 of de Gruyter Studies in Mathematics, Walter de Gruyter, Berlin, Germany, 1993.

[5] I. Laine, J. Rieppo, and H. Silvennoinen, "Remarks on complex difference equations," Computational Methods and Function Theory, vol. 5, no. 1, pp. 77-88, 2005.

[6] J. Rieppo, "On a class of complex functional equations," Annales Academice Scientiarum Fennicee, vol. 32, no. 1, pp. 151-170, 2007.

[7] X.-M. Zheng, Z.-X. Chen, and J. Tu, "Growth of meromorphic solutions of some difference equations," Applicable Analysis and Discrete Mathematics, vol. 4, no. 2, pp. 309-321, 2010.

[8] A. Z. Mokhon'ko, "The Nevanlinna characteristics of certain meromorphic functions," Teorija Funkciǔ, Funkcional'ny̌ Analiz i ih Priloženija, vol. 14, pp. 83-87, 1971 (Russian).

[9] A. A. Mokhon'ko and V. D. Mokhon'ko, "Estimates of the Nevanlinna characteristics of certain classes of meromorphic functions, and their applications to differential equations," Akademija Nauk SSSR, vol. 15, pp. 1305-1322, 1974.

[10] R. Goldstein, "Some results on factorisation of meromorphic functions," Journal of the London Mathematical Society, vol. 4, pp. 357-364, 1971.

[11] R. Goldstein, "On meromorphic solutions of certain functional equations," Aequationes Mathematicae, vol. 18, no. 1-2, pp. 112157, 1978.

[12] G. G. Gundersen, J. Heittokangas, I. Laine, J. Rieppo, and D. Yang, "Meromorphic solutions of generalized Schröder equations," Aequationes Mathematicae, vol. 63, no. 1-2, pp. 110135, 2002. 


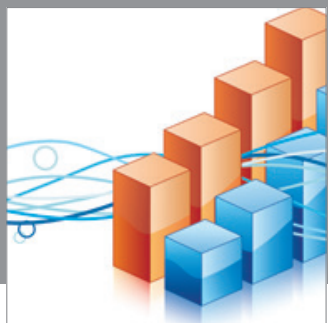

Advances in

Operations Research

mansans

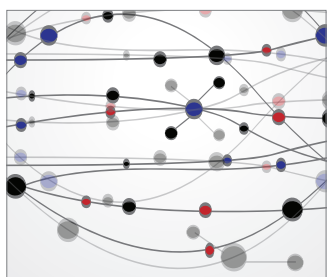

The Scientific World Journal
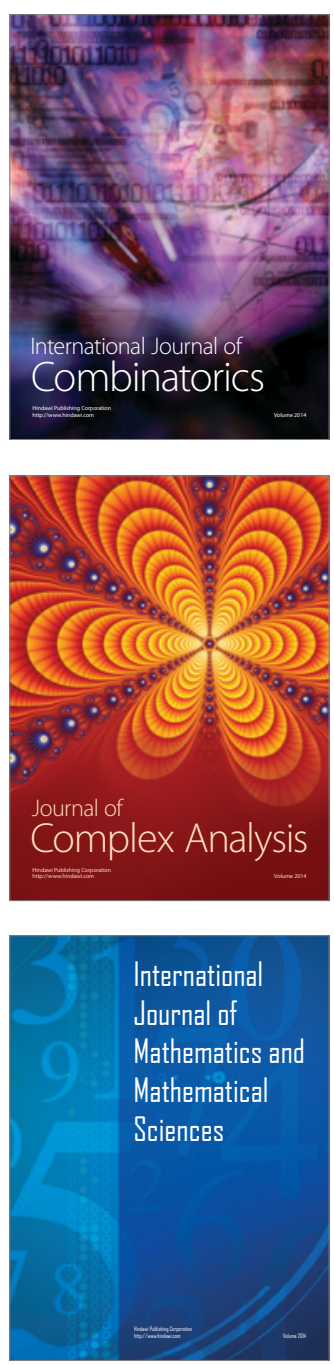
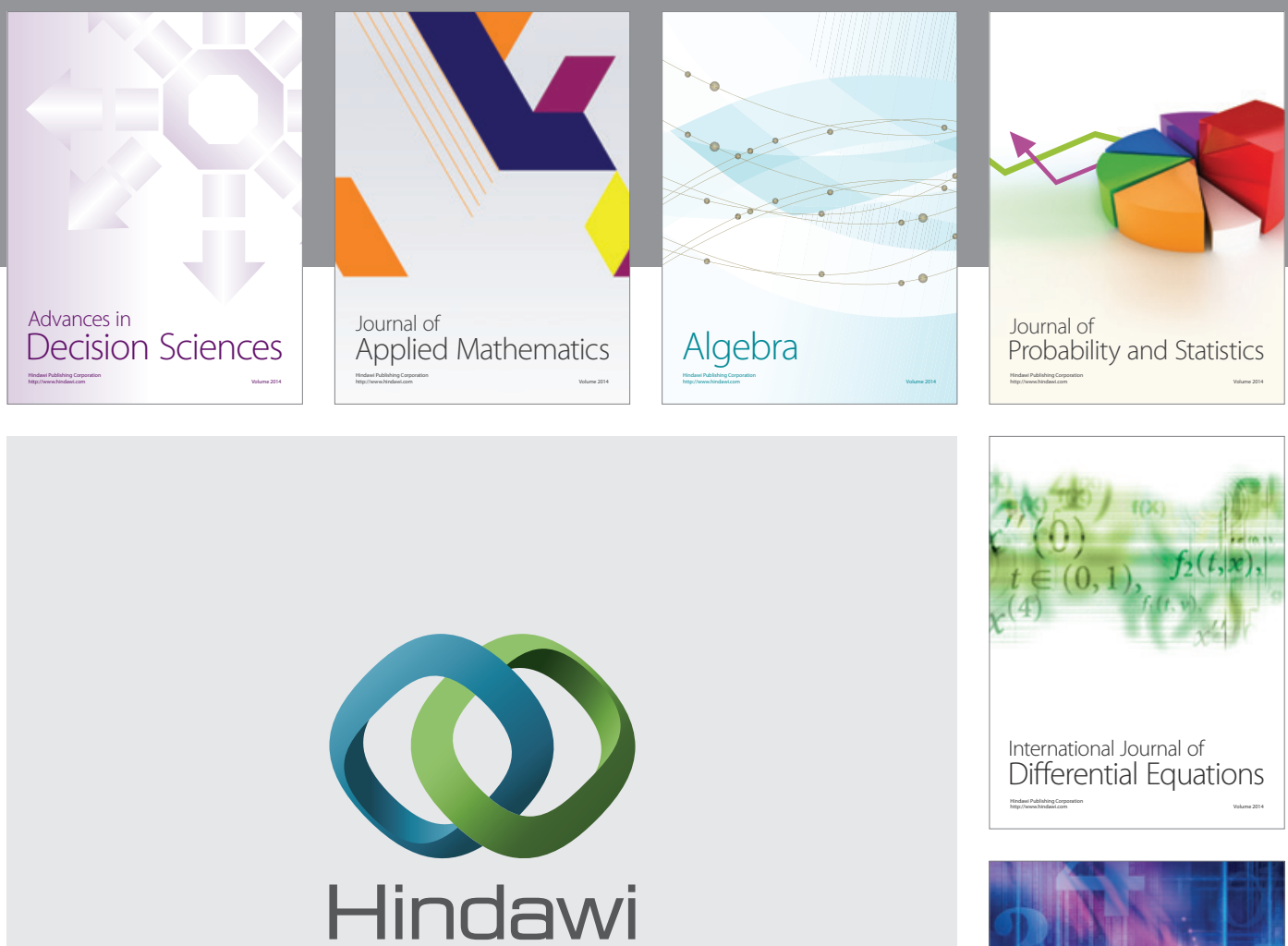

Submit your manuscripts at http://www.hindawi.com
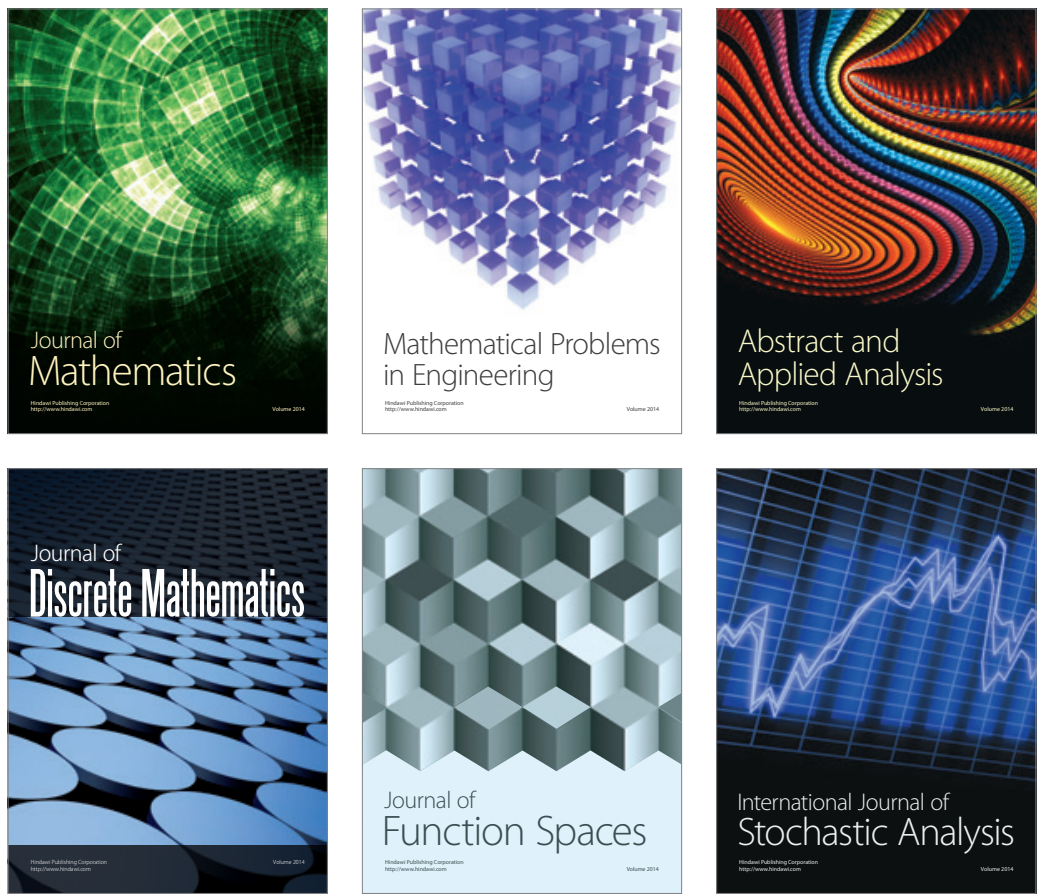

Journal of

Function Spaces

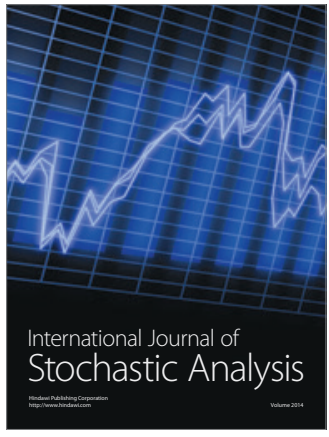

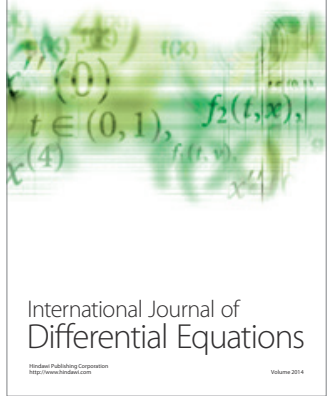
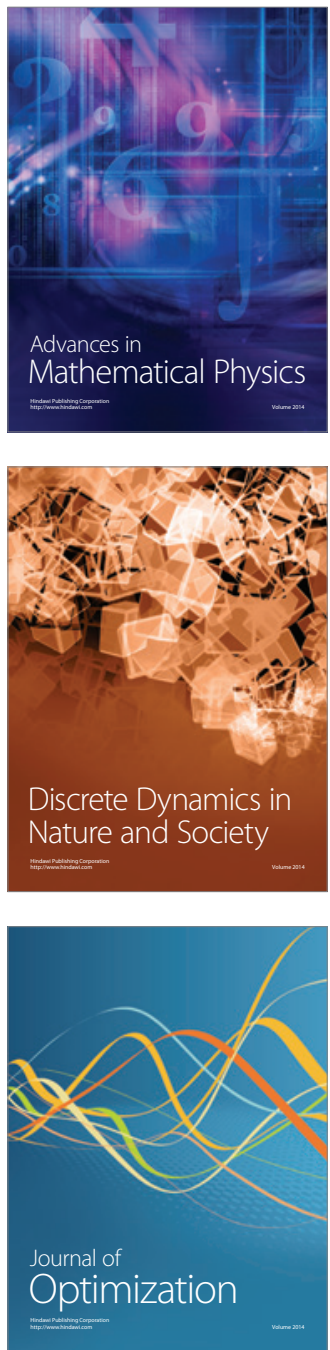